\title{
BLM NP_000048.1:p.Y736fs*4
}

National Cancer Institute

\section{Source}

National Cancer Institute. BLM NP 000048.1:p.Y736fs*4. NCI Thesaurus. Code C131466.

A change in the amino acid sequence of Bloom syndrome protein where a frameshift mutation results in the replacement of a tyrosine residue at position 736 with 4 noncanonic amino acids followed by a stop codon. 